\title{
A Review on Remdesivir: A Possible Promising Agent for the Treatment of COVID-19
}

This article was published in the following Dove Press journal:

Drug Design, Development and Therapy

\author{
Seyed MohammadReza \\ Hashemian (D) 1,2 \\ Tayebeh Farhadi ${ }^{2}$ \\ Ali Akbar Velayati ${ }^{2}$ \\ 'Clinical Tuberculosis and Epidemiology \\ Research Center, National Research \\ Institute of Tuberculosis and Lung \\ Disease (NRITLD), Shahid Beheshti \\ University of Medical Sciences, Tehran, \\ Iran; ${ }^{2}$ Chronic Respiratory Diseases \\ Research Center (CRDRC), National \\ Research Institute of Tuberculosis and \\ Lung Diseases (NRITLD), Shahid \\ Beheshti University of Medical Sciences, \\ Tehran, Iran
}

\begin{abstract}
The novel coronavirus 2019 (2019-nCoV), formally named severe acute respiratory syndrome coronavirus 2 (SARS-CoV-2), is a novel human infectious coronavirus. The disease caused by SARS-CoV-2 is named COVID-19. Development and manufacturing of specific therapeutics and vaccines to treat COVID-19 are time-consuming processes. At this time, using available conventional therapeutics along with other treatment options may be useful to fight COVID-19. In different clinical trials, efficacy of remdesivir (GS-5734) against Ebola virus has been demonstrated. Moreover, remdesivir may be an effective therapy in vitro and in animal models infected by SARS and MERS coronaviruses. Hence, the drug may be theoretically effective against SARS-CoV-2. Remdesivir is a phosphoramidate prodrug of an adenosine $\mathrm{C}$-nucleoside. By entrance into respiratory epithelial cells in human, the prodrug is metabolized to a nucleoside triphosphate as the active form. The nucleoside analog inhibits the viral RNA-dependent RNA polymerase ( $R d R p)$ by competing with the usual counterpart adenosine triphosphate (ATP). The nucleoside analog is incorporated into the generating RNA strand and causes a delayed stop in the viral replication process. Knowledge about the potential efficacy of remdesivir against coronaviruses has been restricted to in vitro studies and animal models. However, information related to COVID-19 is rapidly growing. Several clinical trials are ongoing for the management of COVID-19 using remdesivir. In this study, characteristics of remdesivir and its usage for treatment of COVID-19 are reviewed based on an electronic search using PubMed and Google Scholar.

Keywords: remdesivir, COVID-19, SARS-CoV-2, RNA-dependent RNA polymerase
\end{abstract}

\section{Introduction}

The novel coronavirus 2019 (2019-nCoV), formally named severe acute respiratory syndrome coronavirus 2 (SARS-CoV-2), is a novel human infectious coronavirus. COVID-19 is the disease caused by SARS-CoV-2. The disease was initially found in Wuhan at the end of 2019 and spread rapidly around the world. The number of infectious patients has increased worldwide, resulting in a pandemic situation. ${ }^{1}$

Developing and manufacturing specific therapeutics and vaccines against COVID-19 are time-consuming processes. At this time, administering conventional therapeutics along with other treatment options such as inflammation control, oxygen therapy and fluid management may be valuable to treat the clinical symptoms of the infection such as cough, fever and difficulty breathing. ${ }^{2}$

SARS-CoV-2 shares an $80 \%$ RNA sequence homology with the acute respiratory syndrome-associated coronavirus (SARS-CoV). ${ }^{2,3}$ Some enzymes of both viruses show more than $90 \%$ sequence homology. ${ }^{2}$ For example, RNA-dependent RNA polymerase (RdRp) of both coronaviruses shares $96 \%$ sequence similarity. ${ }^{2}$
Correspondence: Tayebeh Farhadi Chronic Respiratory Diseases Research Center (CRDRC), National Research Institute of Tuberculosis and Lung Diseases (NRITLD), Shahid Beheshti University of Medical Sciences, Tehran, Iran

Tel/Fax +982126109931

Email tayebehfarhadi@yahoo.com 
Accordingly, existing drugs against SARS-CoV and Middle East Respiratory Syndrome coronavirus (MERSCoV) may be also useful to fight COVID-19. ${ }^{1,3}$

In order to target RdRp in the genus Betacoronavirus, some therapeutic options including ribavirin, favipiravir, penciclovir, galidesivir, remdesivir, 6'-fluorinated aristeromycin analogs and acyclovir fleximer analogs are available. ${ }^{4}$ In clinical trials associated with MERS-CoV and Ebola virus infections, remdesivir (GS-5734) has demonstrated to be beneficial. Moreover, using in vitro and in animal models infected by SARS and MERS coronaviruses, remdesivir has shown to be functional. ${ }^{5-11}$ Therefore, the drug may be theoretically effective against SARS-CoV-2.

In this review, the characteristics, activity and usage of remdesivir for treatment of COVID-19 aere discussed. An electronic search was done using databases "PubMed" and "Google Scholar". Key search terms included the association of the following words: "remdesivir", "COVID-19", "SARSCoV-2", "SARS-CoV", "MERS-CoV”, "clinical use", "therapeutic applications", "pharmacodynamics", "pharmacokinetics", "dosing”, "adverse effects", "RNA-dependent RNA polymerase", "2019-nCoV", "Ebola virus", "safety", "mechanism of action", "clinical trial", "diagnosis" and "intravenous administration". Studies without available full-text and studies with title and abstract in English but with a full-text in non-English language were excluded.

\section{Mechanism of Action}

Remdesivir is a phosphoramidate prodrug of an adenosine C-nucleoside (Figure 1A). ${ }^{12}$ By entrance into respiratory epithelial cells in the human body, the prodrug may be efficiently metabolized to a nucleoside triphosphate as an active form (Figure 1B). ${ }^{10}$

The active form can prevent the replication of several coronaviruses in the lung epithelial cells. The nucleoside analog drug inhibits the RNA-dependent RNA polymerase
(RdRp) by competing with the usual counterpart adenosine triphosphate (ATP). The nucleoside analog is incorporated into the generating RNA strand and causes a delayed stop in the viral replication process (Figure 2). ${ }^{10,13}$ As the enzyme incorporates one, two or three more nucleotides, the incorporated nucleoside analog moves back. The drug blocks the enzyme while it reaches into the third position away from the enzyme's active site. It crashes into a conserved serine (Ser) in the active site of the enzyme and inhibits the enzyme from moving one step forward to incorporate the next nucleotide (https://www.asbmb.org/ asbmb-today/science/050720/a-mechanism-for-remdesi vir-activity-and-a-platform) (Figure 2). The exoribonuclease of the virus that usually proofreads and corrects the replication errors cannot work against the active form of remdesivir. ${ }^{2,14}$

\section{Pharmacodynamics and Pharmacokinetics of Remdesivir}

The pharmacokinetics of remdesivir have been summarized in documentation published by the US Food and Drug Administration (FDA). The FDA has issued an emergency use authorization (EUA) to license the emergency use of the unapproved drug remdesivir for management of suspected or laboratory-confirmed SARS-CoV-2 infection in adults and pediatric patients hospitalized with severe disease. ${ }^{15}$

Considering the EUA licensed by FDA, ${ }^{15}$ remdesivir is administered via an intravenous injection (IV) over 30 to 120 minutes. For adult patients, a single loading dose of the drug (200 mg on day 1) followed by once-daily maintenance doses (100 mg from day 2) has been recommended. ${ }^{15}$ For pediatric patients, the dosage of the product should be adjusted for body weight. ${ }^{15}$ For patients requiring invasive mechanical ventilation and/or ECMO and cases not requiring invasive mechanical ventilation and/or ECMO, treatment courses are 10 days and 5 days, respectively. If clinical improvement is not seen in the patients who do not need invasive mechanical
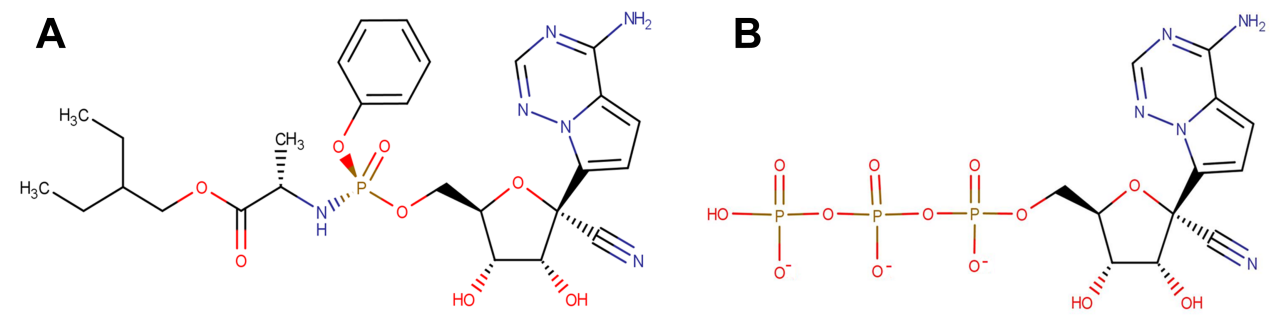

Figure I Chemical structures of (A) remdesivir as the phosphoramidate prodrug of an adenosine C-nucleoside and (B) pharmacologically active nucleoside triphosphate (NTP) as a viral RdRp inhibitor. 


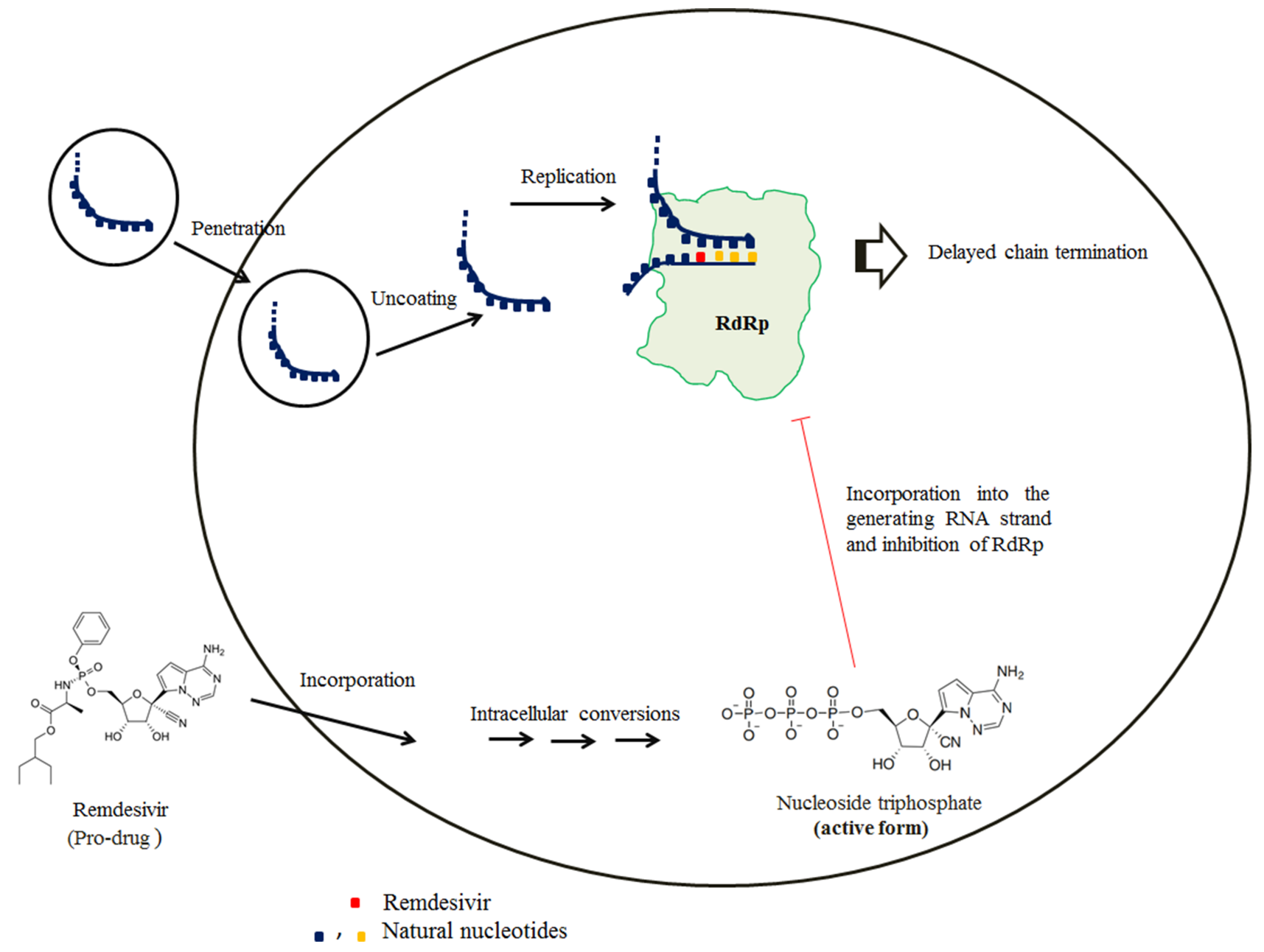

Figure 2 Mechanisms of action of remdesivir. Remdesivir is incorporated into cells and metabolized into the nucleoside triphosphate analog form (active form) via a sequence of steps. The nucleoside analog drug is used by the viral RdRp and inhibits the enzyme by competing with the usual counterpart adenosine triphosphate (ATP). The nucleoside analog is incorporated into the generating RNA strand and causes a delayed stop in the viral replication process.

ventilation and/or ECMO, administration of remdesivir may be continued for up to 5 additional days for a total treatment course of up to 10 days. ${ }^{15}$

However, in a recent trial without a placebo control (ClinicalTrials.gov number: NCT04292899), patients with severe COVID-19 who did not require mechanical ventilation were included. Results did not show any significant difference between the 5-day and 10-day durations of remdesivir administration. Due to the lack of a placebo control arm, the magnitude of remdesivir efficacy cannot be deduced from that trial. The results of the trial suggested that if remdesivir surely is an active therapeutic, supplies that are likely restricted could be saved with shorter courses of remdesivir use. $^{16}$

In cell-based assays, remdesivir was an effective therapeutic against multiple variants of acute Ebola virus and other filoviruses. In a rhesus monkey model of Ebola, after intravenous administration of remdesivir, a lasting level of the nucleotide triphosphate (NTP) was observed in the peripheral blood mononuclear cells $(>10 \mu \mathrm{M})$ for at least $24 \mathrm{~h}$. The half-life of remdesivir was short in blood $(0.39 \mathrm{~h})$, but it was quickly delivered into peripheral blood mononuclear cells and converted into the active drug (half-life $=14 \mathrm{~h}$ ) within $2 \mathrm{~h}$ after administration. The NTP was detected in favorable organs for viral replication such as testes, eyes and brain. ${ }^{10}$ Once-daily intravenous administration of remdesivir $(10 \mathrm{mg} / \mathrm{kg})$ for 12 days blocked the virus replication thoroughly. Administration of remdesivir even after 3 days of the virus exposure protected all infected monkeys against the lethal disease and improved the clinical signs and pathophysiological indicators. $^{10}$

The efficacy of remdesivir against SARS-CoV-2 and associated coronaviruses can be deduced from in vitro 
studies and animal models. For example, using remdesivir for prophylaxis and early treatment in a mouse model with SARS-CoV infection led to a decrease of the viral load in the lungs and improvement of the respiratory function. ${ }^{17}$ In 2020, Wang et al investigated the antiviral efficacy of remdesivir against SARS-CoV-2 in infected Vero E6 cells. The results showed that the IC50 and IC90 values of remdesivir against SARS-CoV-2 were $770 \mathrm{nM}$ and 1760 $\mathrm{nM}$, respectively (cytotoxic concentration $>100 \mathrm{mM}$ ). The authors suggested remdesivir as a highly effective agent against SARS-CoV-2 infection in vitro. ${ }^{18}$

Information obtained from in vitro studies and animal models ${ }^{1017-19}$ along with data obtained from clinical trials on the safety profile of remdesivir against Ebola virus ${ }^{9}$ support remdesivir as a promising agent to fight SARSCoV-2.

\section{Applications of Remdesivir Summary of Antiviral Activity Against Different Viruses}

Remdesivir (GS-5734) was developed by Gilead Sciences. Gilead, the US Centers for Disease Control and Prevention (CDC) and the US Army Medical Research Institute of Infectious Diseases (USAMRIID) collaborated to discover drug candidates against RNA viruses with potential to induce a global pandemic (eg Ebola virus, Middle East respiratory syndrome (MERS) and severe acute respiratory syndrome (SARS) coronaviruses). ${ }^{20-23}$ In order to find suitable antiviral agents against the RNA viruses, a library of approximately 1000 modified nucleosides including monophosphate, ester and phosphoramidate prodrugs was compiled. $^{21-23}$ Results of data screening showed that GS441,524 (a 1'-CN modified adenosine C-nucleoside hit) along with GS-5734 (a prodrug form of the monophosphate of GS-441,524, later renamed as remdesivir) were highly potent antivirals. ${ }^{12}$

In 2016, the first clinical experience of using remdesivir against Ebola virus in human was reported. ${ }^{24}$ Ebola meningoencephalitis was diagnosed in a female nurse by detection of Ebola virus RNA in the plasma and cerebrospinal fluid. The patient was successfully treated by administration of corticosteroids and remdesivir for 2 weeks (once-daily administration of $150 \mathrm{mg}$ over $2 \mathrm{~h}$ for 2 days, and then daily using of $225 \mathrm{mg}$ for 12 days). In spite of a temporary increase in the levels of the enzyme amylase in serum, any serious adverse event was not observed. $^{24}$
In a controlled, randomized clinical trial conducted in the Democratic Republic of Congo, 175 patients were randomly treated with remdesivir for Ebola. Remdesivir was not an effective therapy because of a high rate of death $(53.1 \%)$. However, some data on the safety profile of remdesivir were formally collected. ${ }^{9}$ Results showed that using two other medicines led to superior survival rates compared with remdesivir. Hence, randomization to remdesivir treatment was stopped. ${ }^{25}$ Interestingly, the rate of viral clearance in the subjects who received remdesivir was slower than in the patients who received the single-dose antivirals (specially MAb114 and REGN-EB3). The authors explained that this fact might be associated with a requirement for multiple intravenous infusions of remdesivir. ${ }^{25}$

In 2017, it was shown that remdisiver could prevent the replication of SARS and MERS coronaviruses and be effective against bat and circulating contemporary human coronaviruses in vitro. ${ }^{17}$ In mice, prophylactic and early therapeutic administration of remdesivir significantly decreased the pulmonary viral loads and improved the clinical symptoms of the disease and lung function. It was concluded that remdesivir might be effective against endemic MERS coronavirus, circulating human coronaviruses and, in particular, emerging coronaviruses in the future. ${ }^{17}$

In 2020, de Wit et al studied the prophylactic and therapeutic activities of remdesivir against MERS in rhesus macaques (a nonhuman primate model). Results showed that the viral load in the lungs of the treated animals was lower than that of the controls. The severity of lung lesions in the therapeutic treatment group was lower than that in the vehicle-treated animals. The lungs of the prophylactically treated animals were normal. Taken together, the results emphasized the efficacy of remdesivir as a prophylactic and therapeutic agent against MERS. ${ }^{19}$

In 2020, Sheahan et al showed that remdesivir and interferon-beta have a superior antiviral activity compared to lopinavir and ritonavir against MERS-CoV in vitro. In mice, both prophylactic and therapeutic remdesivir improved the respiratory function and decreased the pulmonary viral loads and severe lung pathology. In contrast, prophylactic lopinavir/ritonavir-interferon beta somewhat decreased the viral loads without affecting other disease factors. Therapeutic lopinavir/ritonavir-interferon beta improved pulmonary function but did not reduce virus replication or severe lung pathology. Overall, the authors suggested that remdesivir might improve the disease outcomes in coronavirus-infected patients, be useful to protect 
health care workers in regions with endemic MERS and inhibit future coronavirus epidemics. ${ }^{7}$

\section{Clinical Efficacy of Remdesivir to Treat COVID-19}

Knowledge about the potential efficacy of remdesivir against coronaviruses is restricted to in vitro studies and animal models; however, information related to COVID19 is rapidly growing. ${ }^{26}$

In January 2020, a 35-year-old male infected with COVID-19 was compassionately treated with intravenous remdesivir after developing pneumonia in the USA. ${ }^{27}$ The patient was hospitalized for more than 12 days, ntravenous remdesivir was administrated for 7 days and the patient's condition was apparently improved on the eighth day. ${ }^{27}$ No adverse effect associated with administration of remdesivir was reported.

Before the clinical trials are completed, information about using remdesivir for COVID-19 can be obtained from studies on compassionate use of the drug. At present, several anecdotal reports on remdesivir administration to treat COVID-19 patients are available. A recent newspaper article reported that using remdesivir to treat 17 passengers on the Diamond Princess cruise ship for 10 days resulted in the patients' survival. Administration of the therapeutic led to less dependence of the patients on a ventilator. However, it should be noted that such reports cannot be considered references for any conclusions. ${ }^{26}$

A preprint reported new medical research on the first 12 COVID-19 patients in the USA; however, authors indicated that the study has yet to be evaluated and so should not be utilized to guide clinical practice. ${ }^{28}$ In the report, demographic and clinical information of the subjects, data on course of infection and clinical care were interpreted. Seven of 12 patients (58\%) with radiographic signs of pneumonia and clinical or laboratory clues of worsening in the second week of the infection were hospitalized. Three hospitalized patients received investigational antiviral remdesivir for 4 to 10 days. In all patients, following remdesivir start, aminotransferase levels were elevated and temporary abdominal symptoms including nausea, vomiting, gastroparesis or rectal hemorrhage were observed. No post-remdesivir symptom was detected. After recovery of the respiratory symptoms, the drug was stopped. $^{28}$

In 2020, in a published study in New England Journal of Medicine, remdesivir was provided on a compassionate-use basis to patients with severe COVID-19. Patients with confirmed SARS-CoV-2 infection and breathing oxygen support or receiving ambient air were included in the study. The oxygen saturation of the subjects was $94 \%$ or less. The drug was administrated for 10 days. At the first day, the patients received $200 \mathrm{mg}$ of the drug intravenously, and $100 \mathrm{mg}$ of remdesivir was daily used in the remaining 9 days of the treatment course. Among 53 patients included in the cohort study, clinical improvement was observed in 36 subjects $(68 \%){ }^{29}$ Data for some patients included in the study were previously described. ${ }^{27,30,31}$

Beigel et al (2020) conducted a double-blind, randomized, placebo-controlled trial on intravenous remdesivir in patients with Covid-19. Patients were randomly divided into treatment and placebo groups receiving remdesivir for up to 10 days. The time of recovery in the patients who received remdesivir was shorter than that of the placebo group (ClinicalTrials.gov number: NCT04280705). ${ }^{32}$

Goldman et al (2020) did a randomized, open-label, phase 3 trial including hospitalized patients with COVID-19. Patients were randomly divided to receive 5-day and 10-day courses of the intravenous remdesivir. In patients with severe Covid-19 not needing mechanical ventilation, there was not any significant difference between the 5-day and 10-day durations of the intravenous remdesivir. Due to lack of a placebo control, the degree of benefit cannot be ascertained (ClinicalTrials. gov number: NCT04292899). ${ }^{16}$

Wang et al (2020) conducted a randomized, doubleblind, placebo-controlled, multicenter trial in adult patients with COVID-19 at ten hospitals in China (ClinicalTrials. gov number: NCT04257656). The 237 patients were randomly divided into the remdesivir (158 cases) and placebo groups (79 cases). Results showed that remdesivir administration is not related to a significant change in the time of clinical improvement. However, patients who received remdesivir had a numerically shorter recovery time compared to those who received placebo. ${ }^{33}$

\section{Clinical Trials}

Several clinical trials of intravenous remdesivir to treat COVID-19 are ongoing. ${ }^{34}$ A double-blinded, placebo-controlled trial (ClinicalTrials.gov identifier: NCT04280705) is underway in the USA in which patients are randomly divided into two placebo and remdesivir groups. In the remdesivir group, patients receive $200 \mathrm{mg}$ of parenteral remdesivir as an initial dose on the first day, and a maintenance dose of the drug (100 mg once-daily) while 
hospitalized for up to 10 days. ${ }^{35}$ The primary outcome of the trial is time to recovery. Day of recovery is the first day on which the patient satisfies one of the following three categories from the ordinal scale: 1) hospitalized, not needing supplemental oxygen - no longer needs ongoing medical care; 2) not hospitalized, limitation on activities and/or needing home oxygen; and 3) not hospitalized, no limitations on activities. The ordinal scale is an assessment of the clinical status at the first assessment of a given study day. The US National Library of Medicine clinical trials registry described the seven category scale as follows: death, hospitalized/on invasive mechanical ventilation or ECMO, hospitalized/on noninvasive ventilation or highflow oxygen devices, hospitalized/necessitating supplemental oxygen, hospitalized/not needing supplemental oxygen, not hospitalized/limited activity, not hospitalized/ no limitations. ${ }^{36}$

A randomized, phase 3 clinical trial of 5 versus 10 days of remdesivir, sponsored by Gilead Sciences, is enrolling patients with severe COVID-19 (ClinicalTrials.gov identifier: NCT04292899). Primary outcomes are the odds of ratio for improvement on a seven-point ordinal scale on day $14 .^{37}$ Another phase 3, randomized trial of remdesivir (ClinicalTrials.gov identifier: NCT04292730) is the same as NCT04292899 except patients enrolling in that study undergo moderate COVID-19. The primary outcome is the proportion of subjects discharged by day $11 .^{38}$

Two double-blinded, placebo-controlled trials recruiting in China were designed to evaluate the efficacy and safety of intravenous remdesivir. ${ }^{39,40}$ One trial (ClinicalTrials.gov

Table I Registered Clinical Trials to Investigate the Efficacy and/Or Safety of Remdesivir for COVID-19

\begin{tabular}{|c|c|c|c|c|}
\hline $\begin{array}{l}\text { Registration } \\
\text { Number }\end{array}$ & Official Title & Status & Country & $\begin{array}{l}\text { Estimated Study } \\
\text { Completion Date }\end{array}$ \\
\hline NCT04257656 & $\begin{array}{l}\text { A phase } 3 \text {, randomized, double-blind, placebo-controlled, multicenter study to } \\
\text { evaluate the efficacy and safety of remdesivir in hospitalized adult patients } \\
\text { with severe COVID-19 }\end{array}$ & Terminated & China & April 10, 2020 \\
\hline NCT04365725 & $\begin{array}{l}\text { A multicenter, retrospective study of the effects of remdesivir in the } \\
\text { treatment of severe COVID-19 infections }\end{array}$ & Recruiting & France & June 2020 \\
\hline NCT04302766 & $\begin{array}{l}\text { An intermediate-size patient population expanded access treatment protocol } \\
\text { for coronavirus disease } 2019 \text { (COVID-19) using remdesivir (RDV; GS-5734 }{ }^{\mathrm{TM}} \text { ) }\end{array}$ & Available & - & - \\
\hline NCT04252664 & $\begin{array}{l}\text { A phase } 3 \text {, randomized, double-blind, placebo-controlled multicenter study to } \\
\text { evaluate the efficacy and safety of remdesivir in hospitalized adult patients } \\
\text { with mild and moderate COVID-19 }\end{array}$ & Suspended & China & April 27, 2020 \\
\hline NCT0432376I & $\begin{array}{l}\text { Expanded access treatment protocol: remdesivir (RDV; GS-5734) for the } \\
\text { treatment of SARS-CoV-2 (CoV) infection }\end{array}$ & Available & - & - \\
\hline NCT044I0354 & $\begin{array}{l}\text { A phase } 2 \text {, randomized, double-blind, placebo-controlled study of the efficacy } \\
\text { and safety of oral merimepodib in combination with intravenous remdesivir in } \\
\text { adult patients with advanced coronavirus disease } 2019 \text { (COVID-19) }\end{array}$ & Recruiting & USA & August 2020 \\
\hline NCT04292730 & $\begin{array}{l}\text { A phase } 3 \text {, randomized study to evaluate the safety and antiviral activity of } \\
\left.\text { remdesivir (GS- } 5734^{\mathrm{TM}}\right) \text { in participants with severe COVID-19 }\end{array}$ & $\begin{array}{l}\text { Active, not } \\
\text { recruiting }\end{array}$ & - & June 2020 \\
\hline NCT04409262 & $\begin{array}{l}\text { A phase } 3 \text {, randomized, double-blind, multicenter study to evaluate the } \\
\text { efficacy and safety of remdesivir plus tocilizumab compared with remdesivir } \\
\text { plus placebo in hospitalized patients with severe COVID-19 pneumonia }\end{array}$ & Recruiting & - & July 31, 2020 \\
\hline NCT0443 I 453 & $\begin{array}{l}\text { A phase } 2 / 3 \text {, single-arm, open-label study to evaluate the safety, tolerability, } \\
\left.\text { pharmacokinetics and efficacy of remdesivir (GS- } 5734^{\mathrm{TM}}\right) \text { in participants from } \\
\text { birth to }<18 \text { years of age with COVID-19 }\end{array}$ & $\begin{array}{l}\text { Not yet } \\
\text { recruiting }\end{array}$ & - & December 2020 \\
\hline NCT04330690 & $\begin{array}{l}\text { A multicenter, adaptive, randomized, open-label, controlled clinical trial of the } \\
\text { safety and efficacy of investigational therapeutics for COVID-19 in hospitalized } \\
\text { patients (CATCO: Canadian Treatments for COVID-19), in conjunction with } \\
\text { the Public Health Emergency SOLIDARITY Trial (World Health Organization) }\end{array}$ & Recruiting & Canada & May 18, 2022 \\
\hline
\end{tabular}


identifier: NCT04252664) targets 308 hospitalized adults with mild-to-moderate COVID-19 and another trial (ClinicalTrials.gov identifier: NCT04257656) targets 452 hospitalized adults with severe COVID-19. In both trials, in a 10-day regimen, the initial dose of remdesivir is $200 \mathrm{mg}$ on day 1 , followed by $100 \mathrm{mg}$ once-daily for remaining days. ${ }^{39,40}$ In both trials, the primary outcome measure is the time to clinical recovery. The time to clinical recovery is defined as the time (in hours) from the start of study medicine (active or placebo) until normalization of fever, respiratory rate, oxygen saturation and alleviation of cough, sustained for at least $72 \mathrm{~h}$, or live hospital discharge, whichever comes first. ${ }^{39,40}$

At this time, there are ten registered clinical trials to investigate the efficacy and/or safety of remdesivir for COVID-19 (Table 1).

\section{Up Going Steps}

Initial findings of different clinical trials are important first steps to develop efficient treatment against COVID-19. ${ }^{41}$ Beigel et $\mathrm{al}^{32}$ published a randomized, placebo-controlled, multicenter study on remdesivir efficacy against SARSCoV-2. The report is significant since it is the first report on the efficacy of remdesivir against a novel coronavirus with unknown pathogenesis. ${ }^{41}$ Results showed that remdesivir has a moderate clinical efficacy against COVID-19. ${ }^{32}$ Findings were preliminary and are followed by more collected data and a full statistical study by all education people. ${ }^{41}$

At this time, several clinical trials have evaluated the efficacy of remdesivir with or without other therapeutics in hospitalized patients with COVID-19. ${ }^{18,29,33}$ As far as we know, there is no registered or published clinical trial focused on evaluating simultaneous administration of remdesivir and dexamethasone in patients on ventilators with COVID-19. The World Health Organization (WHO) has welcomed the initial clinical trial results from the United Kingdom that showed dexamethasone could reduce mortality by one-third in patients on ventilators with COVID-19. For patients requiring only oxygen, administration of dexamethasone decreased the mortality rate by about one-fifth. ${ }^{42}$ Evaluating the efficacy of remdesivir along with dexamethasone in patients on ventilators with COVID-19 may be interesting.

\section{Conclusion}

Repurposing or repositioning available therapeutics is the fastest way to manage a pandemic situation. In vitro and in vivo studies have demonstrated the efficacy of remdesivir against coronaviruses. Moreover, in the current pandemic, some evidence indicates that compassionate use of remdesivir may cause some clinical improvement in patients with COVID-19. ${ }^{29}$

\section{Disclosure}

The authors report no conflicts of interest in this work.

\section{References}

1. Cao YC, Deng QX, Dai SX. Remdesivir for severe acute respiratory syndrome coronavirus 2 causing COVID-19: an evaluation of the evidence. Travel Med Infect Dis. 2020;35:101647. doi:10.1016/j. tmaid.2020.101647

2. Liu W, Morse JS, Lalonde T, Xu S. Learning from the past: possible urgent prevention and treatment options for severe acute respiratory infections caused by 2019-nCoV. Chembiochem. 2020;21(5):730738. doi:10.1002/cbic.202000047

3. Ko WC, Rolain JM, Lee NY, et al. Arguments in favour of remdesivir for treating SARS-CoV-2 infections. Int J Antimicrob Agents. 2020;6.

4. Li G, Cleraq ED. Therapeutic options for the 2019 novel coronavirus (2019-nCoV). Nat Rev Drug Discov. 2020;19(3):149-150. doi:10.1038/d41573-020-00016-0

5. Lo MK, Feldmann F, Gary JM, et al. Remdesivir (GS-5734) protects African green monkeys from Nipah virus challenge. Sci Transl Med. 2019;11(494):eaau9242. doi:10.1126/scitranslmed.aau9242

6. Gordon CJ, Tchesnokov EP, Feng JY, Porter DP, Götte M. The antiviral compound remdesivir potently inhibits RNA-dependent RNA polymerase from Middle East respiratory syndrome coronavirus. J Biol Chem. 2020;295(15):4773-4779. doi:10.1074/jbc. AC120.013056

7. Sheahan TP, Sims AC, Leist SR, et al. Comparative therapeutic efficacy of remdesivir and combination lopinavir, ritonavir, and interferon beta against MERS-CoV. Nat Commun. 2020;11(1):1-4. doi:10.1038/s41467-019-13940-6

8. Warren T, Jordan R, Lo M, et al. Nucleotide prodrug GS-5734 is a broad-spectrum filovirus inhibitor that provides complete therapeutic protection against the development of Ebola virus disease (EVD) in infected non-human primates. Open Forum Infectious Diseases. Vol. 2. Infectious Diseases Society of America; 2015: LB-2.

9. Mulangu S, Dodd LE, Davey RT Jr, et al. A randomized, controlled trial of Ebola virus disease therapeutics. $N$ Engl J Med. 2019;381 (24):2293-2303. doi:10.1056/NEJMoa1910993

10. Warren TK, Jordan R, Lo MK, et al. Therapeutic efficacy of the small molecule GS-5734 against Ebola virus in rhesus monkeys. Nature. 2016;531(7594):381-385. doi:10.1038/nature17180

11. Pedersen NC, Perron M, Bannasch M, et al. Efficacy and safety of the nucleoside analog GS-441524 for treatment of cats with naturally occurring feline infectious peritonitis. J Feline Med Surg. 2019;21 (4):271-281. doi:10.1177/1098612X19825701

12. Siegel D, Hui HC, Doerffler E, et al. Discovery and synthesis of a phosphoramidate prodrug of a Pyrrolo[2,1-f][triazin-4-amino] adenine C-nucleoside (GS-5734) for the treatment of Ebola and emerging viruses. J Med Chem. 2017;60(5):1648-1661. doi:10.1021/acs. jmedchem.6b01594

13. Tchesnokov EP, Feng JY, Porter DP, Götte M. Mechanism of inhibition of Ebola virus RNA-dependent RNA polymerase by remdesivir. Viruses. 2019;11(4):326. doi:10.3390/v11040326

14. Agostini ML, Andres EL, Sims AC, et al. Coronavirus susceptibility to the antiviral remdesivir (GS-5734) is mediated by the viral polymerase and the proofreading exoribonuclease. MBio. 2018;9(2): e00221-18. doi:10.1128/mBio.00221-18 
15. Available from: https://www.fda.gov/media/137566/download. Accessed July 21, 2020.

16. Goldman JD, Lye DC, Hui DS, et al. Remdesivir for 5 or 10 days in patients with severe Covid-19. N Engl J Med. 2020. doi:10.1056/ NEJMoa2015301

17. Sheahan TP, Sims AC, Graham RL, et al. Broad-spectrum antiviral GS-5734 inhibits both epidemic and zoonotic coronaviruses. Sci Transl Med. 2017;9(396):eaal3653. doi:10.1126/scitranslmed.aal3653

18. Wang M, Cao R, Zhang L, et al. Remdesivir and chloroquine effectively inhibit the recently emerged novel coronavirus $(2019-\mathrm{nCoV})$ in vitro. Cell Res. 2020;30(3):269-271. doi:10.1038/s41422-020-0282-0

19. de Wit E, et al. Prophylactic and therapeutic remdesivir (GS-5734) treatment in the rhesus macaque model of MERS-CoV infection. Proc Natl Acad Sci U S A. 2020;117(12):6771-6776. doi:10.1073/ pnas. 1922083117

20. Eastman RT, Roth JS, Brimacombe KR, et al. Remdesivir: a review of its discovery and development leading to emergency use authorization for treatment of COVID-19. ACS Cent Sci. 2020;6:672-683.

21. De Clercq E. Strategies in the design of antiviral drugs. Nat Rev Drug Discov. 2002;1(1):13-25. doi:10.1038/nrd703

22. Mehellou Y, Balzarini J, McGuigan C. Aryloxy phosphoramidate triesters: a technology for delivering monophosphorylated nucleosides and sugars into cells. ChemMedChem. 2009;4(11):1779-1791. doi: $10.1002 / \mathrm{cmdc} .200900289$

23. Seley-Radtke KL, Yates MK. The evolution of nucleoside analogue antivirals: a review for chemists and non-chemists. Part 1: early structural modifications to the nucleoside scaffold. Antiviral Res. 2018;154:66-86. doi:10.1016/j.antiviral.2018.04.004

24. Jacobs M, Rodger A, Bell DJ, et al. Late Ebola virus relapse causing meningoencephalitis: a case report. Lancet. 2016;338(10043):498503. doi:10.1016/S0140-6736(16)30386-5

25. Lo MK, Jordan R, Arvey A, et al. GS-5734 and its parent nucleoside analog inhibit filo-, pneumo-, and paramyxoviruses. Sci Rep. 2017;7 (1):43395. doi:10.1038/srep43395

26. Amirian ES, Levy JK. Current knowledge about the antivirals remdesivir (GS-5734) and GS-441524 as therapeutic options for coronaviruses. One Health. 2020;9:100128. doi:10.1016/j.onehlt. 2020.100128

27. Holshue ML, DeBolt C, Lindquist S, et al. First case of 2019 novel coronavirus in the United States. N Engl J Med. 2020;382(10):929936. doi:10.1056/NEJMoa2001191

28. The COVID-19 Investigation Team. First 12 Patients with Coronavirus Disease 2019 (COVID-19) in the United States. Preprint; 2020.

29. Grein J, Ohmagari N, Shin D, et al. Compassionate use of remdesivir for patients with severe Covid-19. N Engl J Med. 2020;382 (24):2327-2336. doi:10.1056/NEJMoa2007016

30. Kujawski SA, Wong KK, Collins JP, et al. First 12 Patients with Coronavirus Disease 2019 (COVID-19) in the United States. medRxiv; 2020.
31. Lescure FX, Bouadma L, Nguyen D, et al. Clinical and virological data of the first cases of COVID-19 in Europe: a case series. Lancet Infect Dis. 2020;20(6):697-706. doi:10.1016/S1473-3099 (20)30200-0

32. Beigel JH, Tomashek KM, Dodd LE, et al. Remdesivir for the treatment of Covid-19-preliminary report. N Engl J Med. 2020. doi:10.1056/NEJMoa2007764

33. Wang Y, Zhang D, Du G, et al. Remdesivir in adults with severe COVID-19: a randomised, double-blind, placebo-controlled, multicentre trial. Lancet. 2020;395(10236):1569-1578. doi:10.1016/ S0140-6736(20)31022-9

34. U.S. National Library of Medicine Clinical Trials Registry. Coronavirus Trials. Available from: https://clinicaltrials.gov/ct2/ results? cond $=$ Coronavirus $\&$ recrs $=\mathrm{b} \&$ recrs $=\mathrm{a} \&$ recrs $=\mathrm{f} \&$ recrs $=$ d\&age_v $=\&$ gndr $=\&$ type $=\& r s 1 t=\&$ Search $=$ Apply. Accessed July 21, 2020.

35. National Institutes of Health. NIH clinical trial of remdesivir to treat COVID-19 begins. Available from: https://www.nih.gov/newsevents/news-releases/nih-clinical-trial-remdesivir-treat-covid-19begins. Accessed July 21, 2020.

36. U.S. National Library of Medicine Clinical Trials Registry. Adaptive COVID-19 treatment trial. Available from: https://clinicaltrials.gov/ ct2/show/NCT04280705. Accessed July 21, 2020.

37. U.S. National Library of Medicine Clinical Trials Registry. Study to evaluate the safety and antiviral activity of remdesivir (GS-5734 ${ }^{\mathrm{TM}}$ ) in participants with severe coronavirus disease (COVID-19). Available from: https://clinicaltrials.gov/ct2/show/NCT04292899. Accessed July 21, 2020.

38. U.S. National Library of Medicine Clinical Trials Registry. Study to evaluate the safety and antiviral activity of remdesivir (GS-5734 ${ }^{\mathrm{TM}}$ ) in participants with moderate coronavirus disease (COVID-19) compared to standard of care treatment. Available from: https://clinical trials.gov/ct2/show/NCT04292730. Accessed July 21, 2020.

39. U.S. National Library of Medicine Clinical Trials Registry. Mild/ moderate 2019-nCoV remdesivir RCT; 2020. Available from: https://clinicaltrials.gov/ct2/show/NCT04252664. Accessed July 21, 2020.

40. U.S. National Library of Medicine Clinical Trials Registry. Severe 2019-nCoV remdesivir RCT; 2020. Available from: https://clinical trials.gov/ct2/show/NCT04257656. Accessed July 21, 2020.

41. Dolin R, Hirsch MS. Remdesivir-an important first step. $N$ Engl $J$ Med. 2020. doi:10.1056/NEJMe2018715

42. Available from: https://www.who.int/news-room/detail/16-06-2020who-welcomes-preliminary-results-about-dexamethasone-use-in-treat ing-critically-ill-covid-19-patients. Accessed July 21, 2020.

\section{Publish your work in this journal}

Drug Design, Development and Therapy is an international, peerreviewed open-access journal that spans the spectrum of drug design and development through to clinical applications. Clinical outcomes, patient safety, and programs for the development and effective, safe, and sustained use of medicines are a feature of the journal, which has also been accepted for indexing on PubMed Central. The manuscript management system is completely online and includes a very quick and fair peer-review system, which is all easy to use. Visit http://www. dovepress.com/testimonials.php to read real quotes from published authors. 